\title{
Research on Modularization Disassembly Sequence Planning based on Interference Matrix
}

\author{
Yongkai Wei \\ School of Automation, Beijing University of Posts and Telecommunications, Beijing 100876, China \\ yunfei999@126.com
}

Keywords: Disassembly sequence planning, modularization disassembly, combination explosion, hierarchy structure graph.

\begin{abstract}
Disassembly sequence planning is one of the most important works in Product repair. It is very easily cause combinatorial explosion when we disassemble complex machinery product. In order to solve this problem, we put forward modularization disassembly. We construct a modularization disassembly model where the Hierarchy Structure Graph of product is created. Then, we establish an interference matrix on every gradation to record the relationship in product part, and we can get disassembly sequence on every gradation by simplifying the interference matrix. At last, we get whole disassembly sequence by lining up the disassembly sequence on every gradation. Finally, a case study is also given to show that the method is feasible and effective.
\end{abstract}

\section{Introduction}

Products often have to carry out some maintenance operations, and disassembly has a large proportion of product repair, so the research on disassembly sequence planning is very important. From now on, the research on disassembly sequence planning at home and abroad is mainly based on AND/OR Graph[1,2], Directed Graph[3], Undirected Graph[4], Petri Net[5] and Hybrid Graph[6]. These methods can generate disassembly sequence to some extent, but with the increase of parts, the relationship of parts is more and more complex ,and it is very easily cause combinatorial explosion.

To solve this problem, an approach based on the modularization is proposed in the paper to generate the disassembly sequence. This approach gets rid of some parts that don't need to analyze, which decreases the dimension of interference matrix, and avoids combination explosion.

\section{Hierarchy Structure Graph}

In order to avoid combination explosion, we decompose the product to many modules, and then get disassembly sequence by planning modules and parts.

One of the biggest differences between modernization disassembly and general disassembly is that the sequence of modernization disassembly not only contains modules but also part, however, general disassembly only contains parts. The greatest advantage of modernization disassembly is that the parts we need to analyze are decreased. After we choose aim part, we only analyze the parts which have some relationship with aim part. The more parts product has, the better result we can get.

The product is divided to many modules, and some big modules can also be divided to some small models. With the module division on every gradation, we can establish Hierarchy Structure Graph.

Fig. 1 is a Hierarchy Structure Graph. In the Hierarchy Structure Graph, we can find every part in the product. When we choose a part as aim part, we can find the parts and modules which need to analyze from bottom to top. Then, we establish an interference matrix on every floor to get disassembly sequence. At last, we get whole disassembly sequence by lining up the disassembly sequence on every floor from top to bottom. 


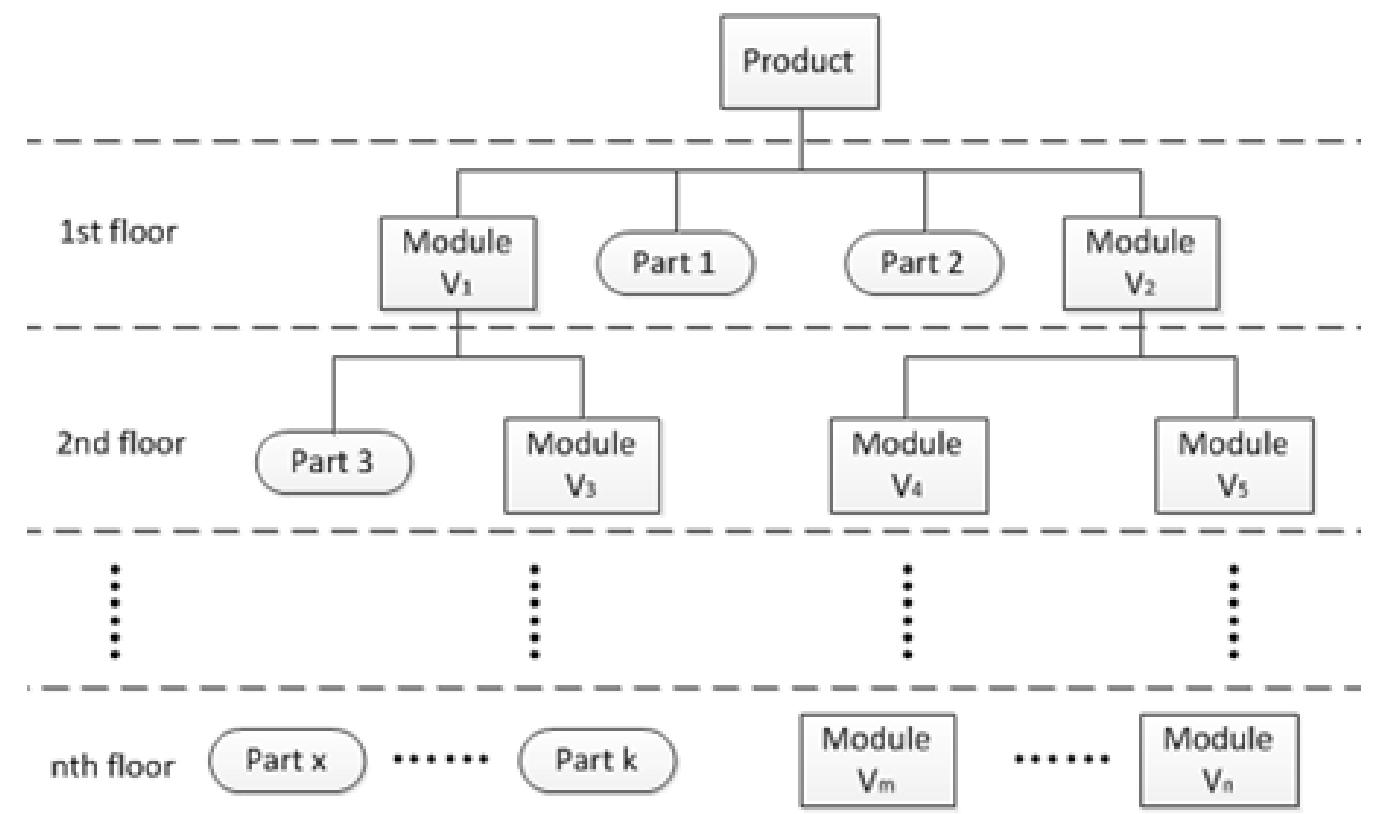

Fig.1 hierarchy structure graph

\section{Disassembly Sequence Planning Based on Interference Matrix}

The definition of part interference : If part $i$ superposes with the fixed part $j$ when it moves in the direction $F(F \in\{+X,-X,+Y,-Y,+Z,-Z\})$, we define that part $i$ interfere with part $j$ in the direction $F$, and mark $M_{i j}(F)=1$; If part $i$ doesn't superpose with the fixed part $j$, we define that part $i$ doesn't interfere with part $j$ in the direction $F$, and mark $M_{i j}(F)=0$; Part doesn't interfere with itself, so $M_{i i}(F)=0$; where, $i, j$ are parts' id.

A product consist of $\mathrm{n}$ parts and there are six disassembly directions $F$ ( $F \in\{+X,-X,+Y,-Y$, $+Z,-Z\})$, so we can get $\operatorname{six} n * n$ matrices, which are called interference matrix. Its expression as below:

$$
M(F)=\left[M_{i j}(F)\right]=\left[\begin{array}{ccc}
M_{11}(F) & \cdots & M_{1 n}(F) \\
\vdots & \ddots & \vdots \\
M_{n 1}(F) & \cdots & M_{n n}(F)
\end{array}\right]
$$

Where, $F \in\{+X,-X,+Y,-Y,+Z,-Z\}$

$I, j=1,2,3 \cdots n$

From the above information, character of interference matrix as follow:

Firstly, when interference matrix has a row whose elements are all 0 , it shows that this row part can be disassembled in this direction.

Secondly, when interference matrix doesn't have a row whose elements are all 0 , it shows that no part can be disassembled in this direction, so we should choose another interference matrix.

Thirdly, there is an interference matrix which has a row whose elements are all 0 at least.

According to the character of interference matrix, we can get disassembly sequence by the algorithm as follow:

Step 1, choose an aim part.

Step 2, choose an interference matrix.

Step 3, check whether interference matrix has a row whose elements are all 0 or not, return to step 2 if NO, or add all 0 row part to disassembly sequence and simplify interference matrix(delete all 0 part's row and column) if YES.

Step 4, repeat step 2 and step 3 till get aim part.

Flowchart is shown in the Fig.2. 


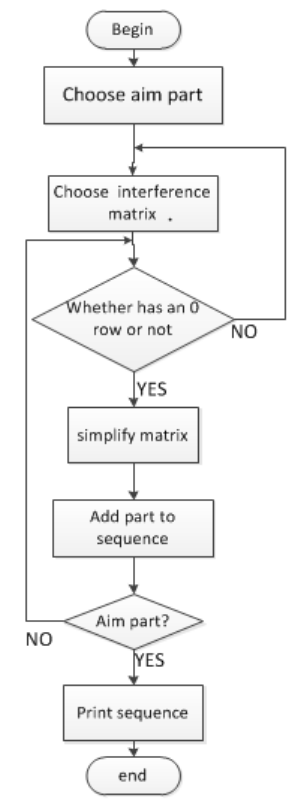

Fig.2 flowchart of algorithm

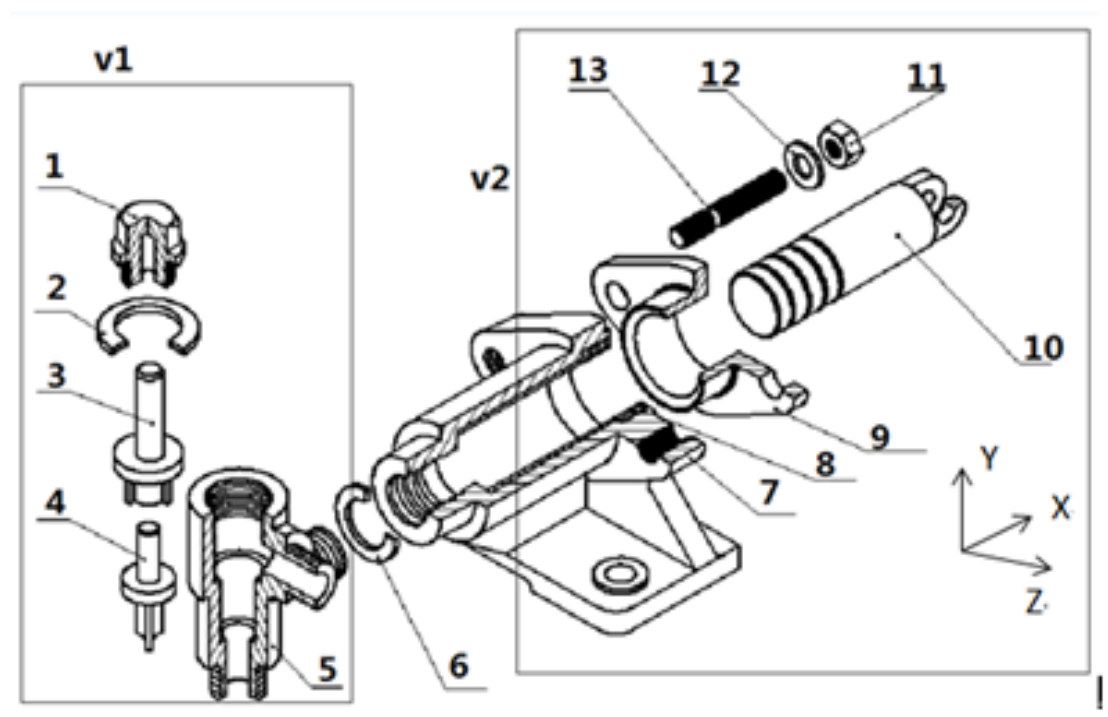

Fig.3 explosive of ram pump

\section{Case Study}

In the Fig.3, it is a ram pump, and we will do modularization disassembly sequence planning research on it.

As shown in the Fig.3, it is an explosive view of ram pump. Product can be divided to two modules on its disassembly direction. Module $V_{1}$ contains part $1,2,3,4,5$ and module $V_{1}$ contains part 7, 8, 9, $10,11,12,13$. The advantage of the module division is that parts' disassembly directions in Module $V_{1}$ are all in "Y" and that in Module $V_{2}$ are all in "X". So we can only establish $M(+Y), M(-Y), M$ $(+X), M(-X)$ to get disassembly sequence. Its Hierarchy Structure Graph is established as follow.

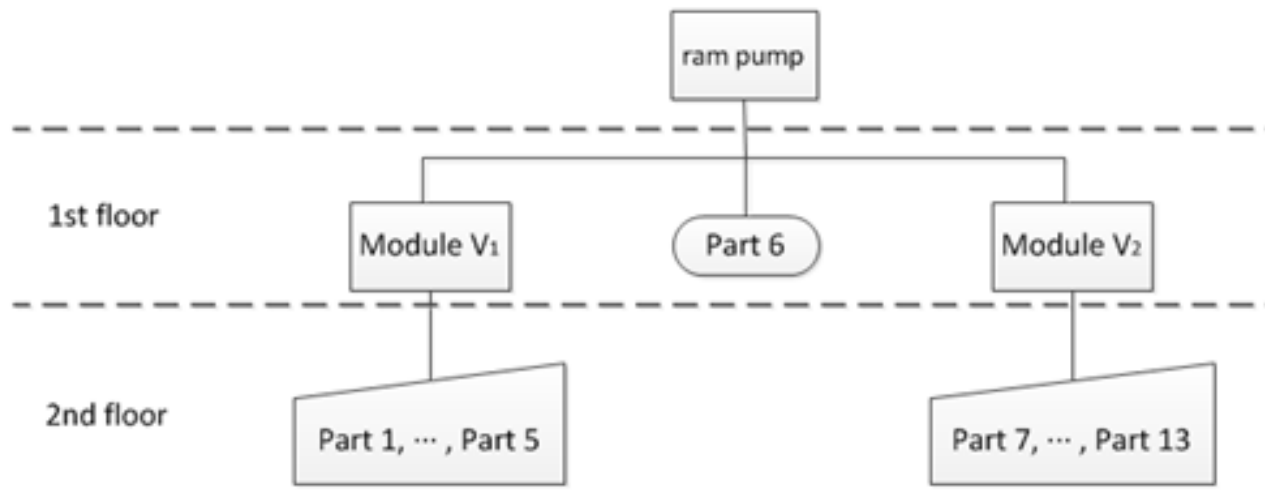

Fig.4 hierarchy structure graph of ram pump

We choose part 4 as aim part and plan its disassembly sequence. In Fig.4, we can find from bottom to top that module $V_{1}$ and part 4 need to be analyzed. Firstly, we establish interference matrix $M_{1}(+\mathrm{X})$ and $M_{1}(-\mathrm{X})$ on 1 st floor, then we can get disassembly sequence of module $V_{1}$ by algorithm. There are two sequences: $V_{1}$ and $V_{2}, 6, V_{1}$; Secondly, we establish interference matrix $M_{2}(+Y)$ and $M_{2}(-Y)$ on 2nd floor, then we can get disassembly sequence of part 4 by algorithm. There are two sequences: 1 , 2, 3, 4 and 1, 5, 4; At last, we line up the disassembly sequence from top to bottom and we can get four sequences. $V_{1}, 1,2,3,4 ; V_{1}, 1,5,4 ; V_{2}, 6, V_{1}, 1,2,3,4 ; V_{2}, 6, V_{1}, 1,5,4$. The number of at least is the best, so the best sequence is: $V_{1}, 1,5,4$. Interference matrix are as follow:

$$
M_{1}(+X)=\left[\begin{array}{lll}
0 & 1 & 1 \\
0 & 0 & 1 \\
0 & 0 & 0
\end{array}\right] \quad M_{1}(-X)=\left[\begin{array}{lll}
0 & 0 & 0 \\
1 & 0 & 0 \\
1 & 1 & 0
\end{array}\right]
$$




$$
M_{2}(+Y)=\left[\begin{array}{lllll}
0 & 0 & 0 & 0 & 0 \\
1 & 0 & 0 & 0 & 0 \\
1 & 1 & 0 & 0 & 0 \\
1 & 1 & 1 & 0 & 0 \\
1 & 1 & 1 & 1 & 0
\end{array}\right]
$$

$$
M_{2}(-Y)=\left[\begin{array}{lllll}
0 & 1 & 1 & 1 & 1 \\
0 & 0 & 1 & 1 & 1 \\
0 & 0 & 0 & 1 & 1 \\
0 & 0 & 0 & 0 & 1 \\
0 & 0 & 0 & 0 & 0
\end{array}\right]
$$

\section{Conclusion}

In this paper, we establish interference matrix to record the relationship between each part and model, and we can get disassembly sequence by simplifying the interference matrix. In order to solve combination explosion problem in complex product, we put forward modularization disassembly. A modularization disassembly model is built with Hierarchy Structure Graph, then, we can get disassembly sequence on every gradation by establishing and simplifying the interference matrix. At last, we get whole disassembly sequence by lining up the disassembly sequence on every gradation. A case study has been proved that this method is feasible and effective.

\section{References}

[1] A.J.D. Lambert, Optimal Disassembly of Complex Products, International Journal of Production Research, 1997, 35(9) p. 2509-2524.

[2] J.R. Li, L.P. Khoo, S.B. Tor, Generation of possible multiple components disassembly sequence for maintenance using a disassembly constraint graph, International Journal of Production Economics, 2006, 23(18) p. 2143-2148.

[3] S.G. Lee, S.W. Lye, M.K. Khoo, A Multi-Objective Methodology for Evaluating Product End-of-Life Options and Disassembly, International Journal of Advanced Manufacturing Technology, 2001, 18(2) p. 148-156

[4] Chulho Chung, Qingjin Peng, An integrated approach to selective-disassembly sequence planning, Robotics and Computer-Integrated Manufacturing, 2005, 21(4) p. 475-485.

[5] Meimei Gao, MengChu Zhou and Ying Tang, Intelligent decision making in disassembly process based on fuzzy reasoning Petri nets, Published in: Systems, Man, and Cybernetics, Part B: Cybernetics, IEEE Transactions on 2004, 34(5) p. 2029-2034.

[6] Xiufen Zhang, Shuyou Zhang and Guodong Yi, Object Selective Disassembly Sequence Planning for Complex Mechanical Products, Journal of Mechanical Engineering, 2010, 46(11) p.172-178. 